University of Texas at El Paso

ScholarWorks@UTEP

$6-2020$

\title{
Preference for Boys Does Not Necessarily Lead to a Gender Disbalance: A Realistic Example
}

Olga Kosheleva

The University of Texas at El Paso, olgak@utep.edu

Vladik Kreinovich

The University of Texas at El Paso, vladik@utep.edu

Follow this and additional works at: https://scholarworks.utep.edu/cs_techrep

Part of the Applied Mathematics Commons

Comments:

Technical Report: UTEP-CS-20-65

\section{Recommended Citation}

Kosheleva, Olga and Kreinovich, Vladik, "Preference for Boys Does Not Necessarily Lead to a Gender Disbalance: A Realistic Example" (2020). Departmental Technical Reports (CS). 1471.

https://scholarworks.utep.edu/cs_techrep/1471

This Article is brought to you for free and open access by the Computer Science at ScholarWorks@UTEP. It has been accepted for inclusion in Departmental Technical Reports (CS) by an authorized administrator of ScholarWorks@UTEP.For more information, please contact Iweber@utep.edu. 


\title{
Preference for Boys Does Not Necessarily Lead to a Gender Disbalance: A Realistic Example
}

\author{
Olga Kosheleva and Vladik Kreinovich \\ University of Texas at El Paso \\ $500 \mathrm{~W}$. University \\ El Paso, TX 79968, USA \\ olgak@utep.edu,vladik@utep.edu
}

\begin{abstract}
Intuitively, it seems that cultural preference for boys should lead to a gender disbalance - more boys than girls. This disbalance is indeed what is often observed, and this disbalance is what many models predict. However, in this paper, we show, on a realistic example, that preference for boys does not necessarily lead to a gender disbalance: in our simplified example, boys are clearly preferred, but still there are exactly as many girls as there are boys.
\end{abstract}

\section{Formulation of the Problem}

Preference for boys - a cultural phenomenon. In many cultures, it is important to have a son. So, if a family has a daughter, the parents continue to produce children until they have the desired son.

In such situations, it is reasonable to expect gender disbalance. Intuitively, it seems that this will lead to a gender disbalance, i.e., that we will have more boys than girls. Such a disbalance is indeed observed in many countries where cultures have such a preference, e.g., in Thailand.

This disbalance is predicted by several models of this phenomenon; see, e.g., [1].

What we do in this paper. In this paper, we consider a simplified model of preference for sons in which, somewhat surprisingly, this preference does not lead to a gender disbalance.

Our main simplifying assumption is based on the fact that in many countries with a strong preference for boys, most people are poor, they cannot afford to have too many children - even one child is not easy to support. For such countries, it is reasonable to make a simplifying assumption that, once the family gets a son, they stop producing children. 
Comment. To make it understandable to people who are interested in demographic questions but may not be mathematically sophisticated, we have tried to make this example as mathematically clear as possible.

\section{Description of Our Example}

Deriving the formula. Let us make an additional simplifying assumption that each new child can be a boy or a girl with equal probability 0.5 , and that genders of different children are statistically independent. In reality, the probabilities of having a boy and having a girl are slightly different form 0.5 , but for our approximate computations, we can ignore this difference.

So, with probability $1 / 2=2^{-1}$, the first child is a son. In this case, according to our assumption, the family will stop producing children. So, in this case, the family will have 0 girls.

If the first child is a girl, then the family produces a second child. With probability $1 / 2$, this second child is a son. Since the genders of different children are statistically independent, the overall probability of this situation is equal to $(1 / 2) \cdot(1 / 2)=2^{-2}$. In this situation, the family has 1 girl.

If the second child is also a girl, then the family produces a third child. With probability $1 / 2$, this third child is a son. Since the genders of different children are statistically independent, the overall probability of this situation is equal to $(1 / 2) \cdot(1 / 2) \cdot(1 / 2)=2^{-3}$. In this situation, the family has 2 girls.

In general, the family can have $n$ girls before they have a boy. The probability of such situation, when we have $n$ girls followed by a boy, is equal to

$$
(1 / 2) \cdot \ldots \cdot(1 / 2)(n \text { times }) \cdot(1 / 2)=2^{-(n+1)} .
$$

In this model, each family has exactly one boy. The expected number $g$ of girls in the family is equal to

$$
g=0 \cdot 2^{-1}+1 \cdot 2^{-2}+2 \cdot 2^{-3}+\ldots+n \cdot 2^{-(n+1)}+\ldots
$$

Computing the formula. Let us find the value $g$. For this purpose, let us multiply both sides of the formula (2) by 2 ; then, each term $n \cdot 2^{-(n+1)}$ becomes

$$
2 \cdot n \cdot 2^{-(n+1)}=n \cdot\left(2 \cdot 2^{-(n+1)}\right)=2^{-n},
$$

so we get

$$
2 \cdot g=0 \cdot 2^{0}+1 \cdot 2^{-1}+2 \cdot 2^{-2}+\ldots+n \cdot 2^{-n}+\ldots
$$

Now, we can subtract, term by term, the formula (2) from the formula (3). Each term in both formulas has the form const $\cdot 2^{-k}$, for some natural number $k$. It is therefore natural to subtract terms corresponding to the same $k$.

- In the formula (2), we have $k=n+1$, so $n=k-1$, and the coefficient at this term is $n=k-1$. 
- In the formula (3), this term corresponds to $k=n$, so the coefficient at this term is $n=k$.

Thus, when we subtract the two expressions, each difference becomes

$$
k \cdot 2^{-k}-(k-1) \cdot 2^{-k}=2^{-k},
$$

so we get:

$$
\begin{gathered}
g=2 \cdot g-g=0 \cdot 2^{0}+(1-0) \cdot 2^{-1}+(2-1) \cdot 2^{-2}+(3-2) \cdot 2^{-3}+\ldots= \\
2^{-1}+2^{-2}+2^{-3}+\ldots+2^{-n}+\ldots
\end{gathered}
$$

To compute the right-hand side of the expression (4), we can use the same trick: double both sides, as a result we get

$$
2 \cdot g=2^{0}+2^{-1}+2^{-2}+\ldots+2^{-(n-1)}+\ldots
$$

When we subtract (4) from (5), all terms $2^{-k}$ cancel each other, expect for the term $2^{0}$ :

$$
g=2 \cdot g-g=2^{0}+\left(2^{-1}-2^{-1}\right)+\left(2^{-2}-2^{-2}\right)+\ldots=2^{0}=1 .
$$

Conclusion. So, for each boy, we have, on average, $g=1$ girl - which shows that there is no gender disbalance, we have exactly as many boys as girls.

\section{Acknowledgments}

This work was supported in part by the National Science Foundation grants 1623190 (A Model of Change for Preparing a New Generation for Professional Practice in Computer Science) and HRD-1242122 (Cyber-ShARE Center of Excellence).

\section{References}

[1] D. Basu and R. de Jong, "Son preference and gender inequality", Proceedings of the Annual Meeting of the Population Association of America AAA'2018, New Orleans, April 17-19, 2008, Section 16. 